\title{
Special issue of the 8th International Workshop on High Aspect Ratio Micro Structure Technology, HARMST 2009
}

\author{
Sven Achenbach • David Klymyshyn • Bernd Michel
}

Published online: 12 June 2010

(C) Springer-Verlag 2010

HARMST 2009 was held from June 25 to 28, 2009, in Saskatoon, Saskatchewan, Canada. This workshop continued a biennial tradition that began in 1995 in Karlsruhe, Germany, and travels around Europe, America, and Asia, successively held in Madison, WI, USA (1997), Chiba, Kisarazu, Japan (1999), Baden-Baden, Germany (2001), Monterey, CA, USA (2003), Gyeongju, Korea (2005), and Besançon, France (2007).

This series has established itself as a premier, independent international event devoted to the advancement of high aspect ratio micro fabrication technologies and their applications. HARMST 2009 again brought together experts from academia and industry from various disciplines and regions who shared their latest results of investigations and developments.

The technical program included sessions on a broad range of aspects of design, modeling, fabrication, metrology, testing, and applications in high aspect ratio micro- and nanotechnology. Particular focus was cast on X-ray and UV-lithography in thick resist layers, using various resist systems and including nonconventional processes, and subsequent electroforming and replication steps, as well as related simulation, modeling, and metrology techniques. Furthermore, selected

S. Achenbach $(\bowtie) \cdot$ D. Klymyshyn

Department of Electrical and Computer Engineering,

University of Saskatchewan, 57 Campus Dr.,

Saskatoon SK S7N 5A9, Canada

e-mail: s.achenbach@usask.ca

S. Achenbach · D. Klymyshyn

TRLabs, 111-116 Research Dr., Saskatoon SK S7N 3R3, Canada

B. Michel

Micro Materials Center Berlin, Fraunhofer IZM,

Berlin, Germany

e-mail: michel@izm.fraunhofer.de; rocks@izm.fraunhofer.de

URL: http://www.springer.com/engineering/electronics/journal/542

advancements in DRIE, mechanical micromachining, and electro discharge micromachining relevant to high aspect ratio technologies were presented, as well as highlights of the integration of nanometer-scale structures, advanced materials, and functionalized surfaces. Applications were another focus of the workshop, from sensors and actuators to RF MEMS, MOEMS, fluidic microsystems, and bio-MEMS. The workshop also included a focus session on challenges and opportunities in micromechanics and microfluidics to discuss a cross section of aspects at the example of a few applications, including industrial needs, fabrication technology capabilities and restrictions, metrology, and testing.

HARMST 2009 featured 74 poster and 39 oral presentations from 15 countries as well as 8 short course contributions.

The HARMST workshops have been highly successful and continue their tradition not only in terms of presentation and discussion during the workshop, but also with respect to the timely dissemination of cutting edge technologies via the Journal of Microsystem Technologies. This Special Issue of Microsystem Technologies is collecting 53 finally accepted contributions as reviewed and unabridged versions of papers presented at the workshop, following the traditional cooperation between Springer and the HARMST Technical Advisory Committee. We hope that you will enjoy reading the HARMST contributions as much as we did.

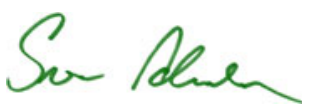

Sven Achenbach

HARMST 2009 Chair and Guest Editor,

HARMST 2009 Special Issue of MST

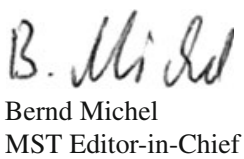

MST Editor-in-Chief
David Klymyshyn HARMST 2009 Co-Chair

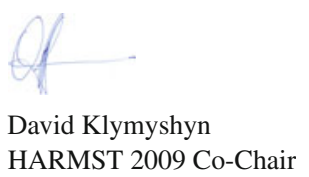

